
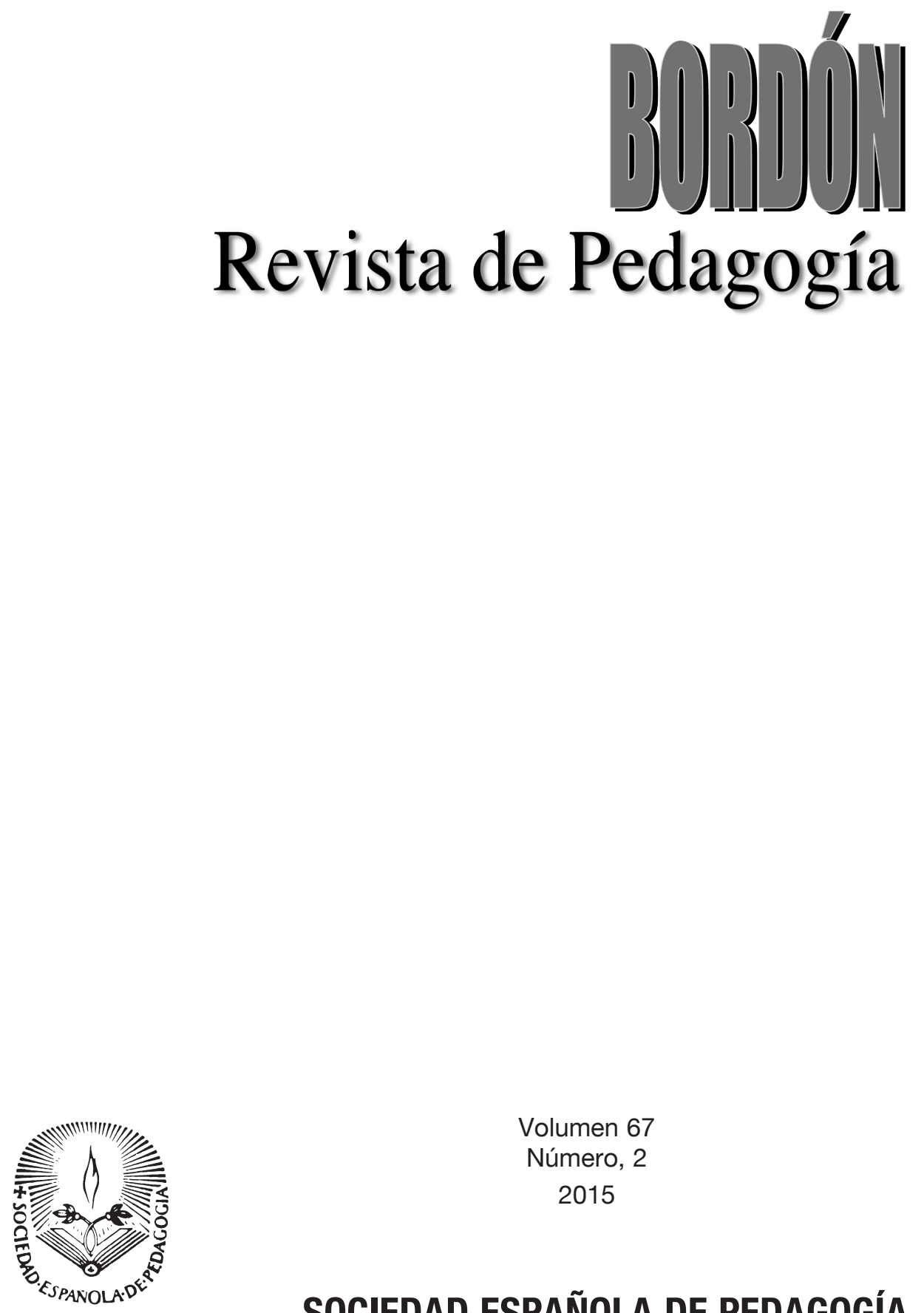

Volumen 67

Número, 2

2015

SOCIEDAD ESPAÑOLA DE PEDAGOGÍA 


\title{
LAS FUNCIONES DEL EDUCADOR SOCIAL: UALIDACIÓn DEL CUESTIONARIO CFES-R
}

\section{The role of the social educator: validation of the CFES-R questionnaire}

\author{
JOSEP VALLÉS HERREROY RAMÓN PÉREZ JUSTE \\ Universidad Nacional de Educación a Distancia (UNED)
}

D0I: 10.13042/Bordon.2015.67209

Fecha de recepción: 01/12/2013 - Fecha de aceptación: I6/10/2014

Autor de contacto / Corresponding Author: Josep Vallés Herrero. Email: jvalles@tortosa.uned.es

INTRODUCCIÓN. En este artículo se presenta el cuestionario sobre las funciones del educador social, CFES-R, como instrumento útil y adecuado para el estudio, evaluación e investigación en torno a las funciones genéricas de esa profesión. Su contenido recoge aquellas funciones educativas genéricas que ejercen los profesionales de forma efectiva en pro del bienestar individual y social de los menores vulnerables y personas excluidas socialmente o en riesgo de exclusión. Por otra parte, y dada la necesaria relación entre la formación académica recibida y la capacitación para el ejercicio competente de las funciones profesionales, se aborda, mediante una tabla de especificaciones, su posible utilización como instrumento para la valoración de las mismas. MÉTODO. Se ha llevado a cabo una investigación descriptiva y correlacional, con una muestra estatal de 1.242 educadores, en 16 comunidades autónomas. El instrumento, para cuya elaboración y validación se ha acudido a la teoría clásica de los tests, ha sido construido a partir del desarrollo de un modelo teórico basado en el acompañamiento terapéutico o reeducativo a los beneficiarios, diferenciando tres subescalas de funciones: orientadoras, comunitarias y de gestión. RESULTADOS. El cuestionario alcanza una elevada fiabilidad, medida con la prueba alfa de Cronbach, y cuenta con suficiente validez de contenido, obtenida a partir de documentos profesionales y a través de paneles de expertos. La fiabilidad global es de .94 y la de sus subescalas integrantes (orientación personal, trabajo comunitario y de gestión) en torno al .90. DISCUSIÓN. Los 37 ítems del instrumento presentan mediciones estables y consistentes. Las funciones en torno al acompañamiento reeducativo son relevantes y se demuestra la existencia de la subescala de funciones comunitarias. Requieren de estudios de mayor profundidad las otras subescalas (orientación y gestión). Puede ser un instrumento muy útil en la valoración de la formación académica e, incluso, en la revisión y mejora de los planes de estudio.

Palabras clave: Descripciones de trabajo, Funciones, Educadores, Educación comunitaria, Cuestionarios, Fiabilidad, Validez, Terapéutico. 


\section{Introducción}

En España, la titulación de diplomado en Educación Social se concibió como una enseñanza de primer ciclo universitario dirigida a la formación de una figura profesional en el campo de la educación no formal, educación permanente y de adultos, inserción social de las personas desadaptadas y con discapacidad, así como en las distintas acciones socioeducativas (Real Decreto 1420/1991 del Ministerio de Educación).

Recientemente, las universidades se han visto en la necesidad de introducir cambios para adaptar sus planes de estudio al Espacio Europeo de Educación Superior (EEES), como reflejó en el año 2007 el monográfico "Pedagogía Social y convergencia europea", de la Revista Interuniversitaria-Pedagogía Social, orientando sus estudios a la adquisición de competencias adecuadas para el ejercicio responsable de una profesión (Guerrero y Calero, 2013), si bien estos cambios no han terminado de implantarse aunque se están realizando esfuerzos para corregir tales disfunciones (Senra, 2012).

En sintonía con esta nueva manera de concebir la formación hemos llevado a cabo una investigación centrada en la dimensión profesionalizadora de los graduados a partir de la entrada en vigor del EEES, que reivindica la necesidad de que la universidad esté atenta a la complejidad de los contextos laborales, que establezca puentes con los profesionales mediante estudios empíricos que contribuyan a la justificación de unas funciones genéricas que permitan un perfil profesional de espectro amplio, con independencia del contexto laboral en que se trabaje.

Funciones del educador social en el marco de la profesionalización

Desde hace más de una década, especialmente a partir de la creación de la diplomatura, la intervención profesional viene planteándose con una gran variedad de enfoques y tratamientos que, a su vez, se encuentran integrados en el refuerzo de las competencias como base fundamental de la profesionalización; a estos planteamientos se ha llegado tanto desde el punto de vista de las asociaciones y colegios profesionales de los educadores sociales, como la Asociación Estatal de Educadores Sociales (ASEDES), la Asociación Internacional de Educadores Sociales (AIEJI) y el Consejo General de Colegios de Educadoras y Educadores Sociales de España (CGCEES), que sustituye a la anterior ASEDES, como desde los principales teóricos de la Pedagogía Social, entre los que destacamos las obras de Petrus (1997), Quintana (2002), Pérez (2004), Caride (2005) y Sáez y García (2006).

Desde la perspectiva del perfil del educador social, hay estudios que aportan algunas claves respecto al trabajo de educadores; destacamos los de Múgica (1991), Petrus (1993), López de Aróstegi (1995), Santolaria (1997), Romans (2000), Tiana y Sanz (2003), Campillo, Bas y Sáez (2004), Vallés (2009), Moyano (2012) y Varela (2012).

En nuestro caso, se trata de identificar, analizar y valorar funciones educativas genéricas que trabajen de forma efectiva por el bienestar individual y social de los menores vulnerables y personas excluidas socialmente o en riesgo de exclusión. Si bien no son muchas las investigaciones sobre este tema, contamos con análisis teóricos sobre esta cuestión y sobre su relación con la profesionalización (Caride, 2002; Korncbeck, 2002; Planella, 2003; Sáez, 2003; Scarpa y Corrente, 2007; Arandia y otros, 2012 y, especialmente, los editados en relación a Europa por la Oficina Internacional del CGCEES, de Calderón y Gotor, 2013). Tras la consulta de estos documentos, parece que lo que tienen en común los educadores y las educadoras sociales de todo el mundo son una serie de funciones sobre las que hemos querido investigar tratando de comprobar, en nuestro país, el grado de generalidad de las mismas; esta hipótesis no puede verificarse en los escasos documentos de 
investigación internacional, como se desprende de búsquedas con ERIC y PROQUEST; con estas bases de datos, a través de los parámetros "functions AND educators AND (educators, questionnaires)" se producen 29 resultados. Seleccionamos, por el "abstract", los artículos de Green y Barnes (1989), Utley y Rapport (2002), Dixon, Meier, Brown y Custer (2005), Petitpierre, Lobo, Dietrich, Benz y Adler (2007), Koster y Dengerink (2008), Kenaszchuk, Rykhoff, Collins, McPhail y Van-Soeren (2012). Bajo tales parámetros de búsqueda, aparecen unos perfiles profesionales poco estudiados mediante cuestionarios, además de responder a diferentes denominaciones y en ocasiones sin formación universitaria (p. ej., educadores especializados y auxiliares docentes). Nos sorprende que, a partir de la palabra clave "educator" recogida en ERIC, no aparezcan estudios donde se mencionen otras denominaciones profesionales similares, como los educadores profesionales italianos, los psicoeducadores del Canadá o los pedagogos sociales alemanes.

En el artículo de Koster y Dengerink (2008) se presenta la norma holandesa SPR para formadores de docentes como un instrumento para el desarrollo profesional que debe ir actualizándose cada cuatro años. En los recientes debates con los formadores de docentes de otros contextos nacionales se plantea como preferible el marco profesional al de las normas.

Dixon y otros (2005) abordan la importancia de las competencias desempeñadas en el mercado laboral para la formación de profesionales en Jamaica.

El estudio de Ruby (2012) trata un aspecto tan original como llamativo, pues centra su atención en las decisivas funciones de los abuelos, como educadores eficaces que combinan el afecto y el objetivo de una enseñanza compleja como es el idioma materno.

Por su parte, Green y Barnes (1989) analizan mediante un instrumento de evaluación el papel de auxiliares docentes que históricamente ejercía el clero. La relevancia del trabajo colaborativo como una característica de las relaciones de trabajo efectivas entre los profesionales de educación especial y servicios relacionados en el ámbito educativo se estudia en el artículo de Utley y Rapport (2002).

Las actitudes de colaboración interprofesional son objeto de estudio analizando unos talleres interprofesionales, en Toronto (Canadá), dirigidos a asistentes sociales y estudiantes de salud por Kenaszchuk y otros (2012). Por su parte, Petitpierre y otros (2007) estudiaron en Alemania y Suiza cómo se organiza coordinadamente la prestación de atención y apoyo educativo y terapéutico para niños con profundas discapacidades múltiples.

De todas estas aportaciones parece deducirse la necesidad de la adecuación de la formación al mundo laboral, además de la importancia del trabajo en equipo. Por otra parte, desde esta perspectiva, nuestro planteamiento centrado en la identificación de las funciones profesionales en España nos permitirá valorar la formación académica recibida por los educadores sociales.

\section{La génesis del CFES-R (Cuestionario de Funciones del Educador Social Revisado)}

Nuestro cuestionario se enmarca en una investigación concebida para contribuir a evitar el "síndrome del caballero andante" (Vallés, 2009), es decir, el de aquel profesional, educador social en este caso, que trabaja en diversos espacios de intervención y no sabe exactamente a qué funciones atenerse al tratarse de una profesión relativamente reciente en nuestro país y al ocupar un espacio profesional creado e institucionalizado a partir de unas necesidades socioeducativas concretas, pero al que llega después de otros profesionales más asentados laboralmente.

La génesis del CFES-R se encuentra en un trabajo previo en el que analizamos las funciones 
de los educadores sociales en los servicios sociales de Cataluña ${ }^{1}$ mediante el CFES-R 2008. En esta ocasión se amplía el estudio a la mayoría de áreas de intervención de los educadores: social, discapacidad, mayores y menores; por otra parte, el estudio se extiende a todas las comunidades autónomas de España, excepto Cantabria, por carecer entonces de colegio o asociación profesional.

El CFES-R es el resultado de un proceso destinado a profundizar en sus características técnicas, en especial en su fiabilidad y validez de contenido. Es un trabajo singular porque la encuesta ha sido aplicada a educadores que están ejerciendo y tienen un contrato como tales, siendo el primero que centra su objetivo principal en las funciones de los educadores sociales en España.

El cuestionario se centra en el estudio de las funciones genéricas de los educadores sociales, esto es, las tareas y trabajos concretos que delimitan un ámbito de responsabilidad laboral que el profesional es capaz de realizar con cierto nivel de garantía (ASEDES, 2006). A partir de esta idea, nuestro objetivo fue diseñar y validar un instrumento que permitiera identificar las funciones profesionales que ejerce realmente un educador social.

Cabe pensar que tal competencia para el ejercicio profesional tiene como base los aprendizajes significativos adquiridos a través de la formación orientada a tales actividades profesionales. Como consecuencia, nuestro planteamiento sobre la relación entre funciones y competencias es el recogido a continuación:

Aprendizajes significativos (producto de la formación académica) $\rightarrow$ Funciones (en relación a un contexto laboral y tareas) $\rightarrow$ Competencias profesionales (saber, saber hacer y saber estar).

Este planteamiento encuentra apoyos en la definición de educación social de ASEDES $(2004)^{2}$, que establece el punto de partida para pensar y concretar las funciones generales del educador social. En este sentido, definimos como genéricas las funciones que se dan en la mayoría de contextos laborales, apoyándonos fundamentalmente en Cacho (1996) ${ }^{3}$. Para la concreción de las funciones que proponemos en el cuestionario de validación, hemos tenido en cuenta, además, las siguientes propuestas:

- Los inventarios de funciones y ámbitos de intervención que hicieron autores como Múgica (1991), Petrus (1993), López de Aróstegui (1995) y Romans (2000).

- La normativa en Cataluña de los Servicios Sociales (La Ley 4/94 de la Generalitat y los decretos 17/94 y 284/96).

- La propuesta sobre funciones de la Comisión de Expertos de la Generalitat de Cataluña sobre profesionales de los Servicios Sociales de Base (1996).

- El catálogo de las funciones explicitadas por el Catálogo de Funciones y Competencias (2006) elaborado por una Comisión de Expertos de la ASEDES.

Con estas aportaciones definimos al educador como un profesional cuyas funciones derivan de la finalidad de ayuda en el proceso de desarrollo personal y socialización de los beneficiarios de su intervención. En este proceso, en coherencia con nuestra base teórica, preguntamos a los educadores sociales, con vinculación laboral, sobre las funciones que ejercen, agrupadas en los tres bloques o ámbitos que mostramos en la figura 1 y que se convertirán en dimensiones en el cuestionario, con su propia especificidad.

Figura 1. Funciones profesionales del educador social Funciones profesionales Ámbito de incidencia

\begin{tabular}{ll} 
Orientadoras & Personal y familiar \\
\hline Comunitarias & Grupal \\
\hline De gestión & Institucional
\end{tabular}


El cuestionario tiene como referencia un modelo teórico, el presentado en la figura 2. En el centro encontramos la esencia o naturaleza de la educación social, la ayuda, que consiste en el acompañamiento reeducativo y terapéutico. Este es, según nuestra investigación empírica, el núcleo central de la polivalencia que permite reflexionar sobre las otras funciones, de orientación, comunitarias y de gestión que se hallan en la naturaleza de la acción del educador, y en la relación que establece con los sujetos de la educación más allá de lo escolar y de la formación reglada.

Figura 2. Esencia y funciones polivalentes de los educadores sociales

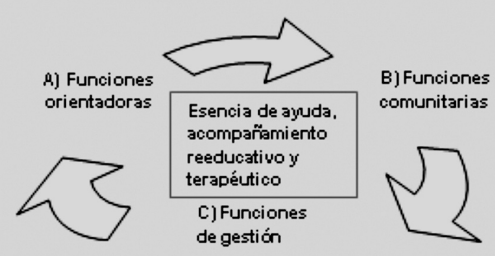

\section{Metodología}

\section{Diseño}

Atendiendo a las características de nuestro trabajo, en particular al tipo y cantidad de información a recoger, número de sujetos a los que acudir y aspectos como su dispersión y diversidad, elegimos la metodología de encuesta frente a otras técnicas como la observación, participante o no, las entrevistas o los grupos focales.

La elaboración del CFES-R se orientó a constatar empíricamente, con métodos y técnicas propios de la teoría clásica de los tests (TCT) y de un estudio descriptivo y correlacional mediante técnica de encuesta, si es posible hablar con el debido rigor de funciones genéricas de los educadores sociales, es decir, de aquellas comunes a cualquier contexto laboral ${ }^{4}$. A tal efecto, hemos construido el modelo teórico, ya presentado en la figura 2, para elaborar la estructura del cuestionario, esperando que de las respuestas recogidas, podamos inferir la realidad sobre las funciones desempeñadas. El análisis de datos ha sido fundamentalmente cuantitativo habiéndose aplicado técnicas descriptivas multivariantes, en concreto un análisis factorial exploratorio destinado a conocer la estructura factorial del cuestionario. De gran importancia han resultado los análisis de validez a través del panel de expertos y de fiabilidad (alfa de Cronbach). Además se ha examinado el Index of Fit for Factor Scales (IFFS) de Fleming que permite valorar la relevancia de las subescalas.

\section{Instrumento}

A partir de este modelo se elaboraron los ítems integrantes del CFES en su versión inicial para expertos, que evolucionó en el CFES-R 2008, para los servicios sociales de base, y en el definitivo CFES-R 2009, con 37 ítems, siguiendo las fases propuestas por Sierra (1988: 306): formulación de objetivos, fijación de dimensiones, variables e indicadores y planificación del contenido del cuestionario. Organizamos las funciones en tres grandes bloques: el de funciones orientadoras (ítems 1-8), el de las comunitarias (ítems 9-19), que consideramos socioeducativas, y el de las de gestión (ítems 20-37), que catalogamos como instrumentales, al servicio de las anteriores.

\section{Muestra, población y aplicación del instrumento}

El cuestionario se aplicó a una muestra de sujetos, seleccionados mediante muestreo estratificado por comunidades autónomas (tabla 1). Fueron los colegios profesionales de las comunidades autónomas los que distribuyeron entre sus colegiados los cuestionarios que les fueron 
asignados y llevaron a cabo la reposición de los no presentados.

El muestreo, con la colaboración del Consejo General de Colegios de Educadoras y Educadores Sociales de España —CGCEES—, es estatal y representa a nuestra población de estudio - los educadores y las educadoras- segmentada en colegios o asociaciones profesionales por comunidades autónomas. Elegimos este muestreo al estar la población de estudio dividida en colegios o asociaciones profesionales que se

TABLa 1. Distribución y error estimado del muestreo obtenido

Muestreo

\begin{tabular}{|c|c|c|c|c|}
\hline & & Colegiados & Deseado & $\begin{array}{c}\text { Obtenido } \\
01 / 05 / 2010\end{array}$ \\
\hline CEESG & Galicia & 1.070 & 102 & 106 \\
\hline CEESC & Cataluña & 3.800 & 364 & 359 \\
\hline CEESIB & Baleares & 493 & 47 & 78 \\
\hline CESCLM & Castilla-La Mancha & 514 & 49 & 82 \\
\hline CEESCYL & Castilla León & 652 & 63 & 68 \\
\hline CEESAragón & Aragón & 786 & 75 & 31 \\
\hline GHEE-CEESPV & País Vasco & 493 & 47 & 68 \\
\hline COEESCV & Valencia & 1.110 & 106 & 55 \\
\hline CPESRM & Murcia & 610 & 58 & 55 \\
\hline COPESA & Andalucía & 1.420 & 136 & 89 \\
\hline APESPA & Asturias & 50 & 5 & 5 \\
\hline ACAES & Canarias y Tenerife & 58 & 6 & 6 \\
\hline APESC & Canarias y Tenerife & 52 & 5 & 5 \\
\hline APES-NAVARRA & Navarra & 94 & 9 & 11 \\
\hline APES LA RIOJA & Rioja & 40 & 4 & 14 \\
\hline AMES & Madrid & 222 & 21 & 61 \\
\hline AEXES & Extremadura & 150 & 14 & 49 \\
\hline TOTAL & & 11.614 & 1.111 & 1.242 \\
\hline \multicolumn{5}{|c|}{ Muestreo real inferior al ideal } \\
\hline \multicolumn{5}{|c|}{ Muestreo real superior al ideal } \\
\hline \multicolumn{5}{|l|}{ A nivel global } \\
\hline \multicolumn{5}{|c|}{ Error para $95 \%$ de NC 2,6\% } \\
\hline \multicolumn{5}{|c|}{ Error para $97 \%$ de NC $2,9 \%$} \\
\hline \multicolumn{5}{|c|}{ Aragón Valencia Andalucía } \\
\hline \multicolumn{5}{|c|}{ Error para $95 \%$ de NC $17,3 \% 12,9 \% 10,1 \%$} \\
\hline Error para $97 \%$ de & & & & \\
\hline
\end{tabular}


suponen con cierta variación con respecto a las opiniones sobre las funciones o características que se van a estudiar. A cada uno de estos estratos se les asignó una cuota que determinó el número de miembros del mismo que supondría la muestra ideal a conseguir.

Los cuestionarios fueron enviados a una muestra de 1111 educadores sociales y distribuidos dentro de cada comunidad autónoma que actuaba como estrato, con asignación proporcional al número de sus profesionales; el valor citado se obtiene a partir de la tabla de Tagliacarne, para $\mathrm{p}=\mathrm{q}(50 / 50)$, con un margen de error del 3\% y un nivel de confianza del $95.5 \%$ $(2 \sigma)$. El cuestionario lo dirigimos a educadores en las áreas de actuación seleccionadas (Social, Menores, Discapacitados y Personas Adultas). Tras la reposición de los no implementados los colegios profesionales remitieron un total de 1242 cuestionarios, lo que supone un margen de error del 2,6\% y un nivel de confianza del $95,5 \%(2 \sigma)$.

\section{Resultados}

La calidad de un instrumento de recogida de datos depende fundamentalmente de su grado de validez y fiabilidad. En relación con la primera se ha tratado de una validez de contenido establecida por el procedimiento de juicio de expertos. En cuanto a la fiabilidad, ha sido calculada mediante el alfa de Cronbach.

\section{Aproximación a la estructura factorial del cuestionario}

Además del estudio sobre la validez y fiabilidad se han realizado otros de interés, destacando los relativos a las correlaciones ítem-total, que presentamos más adelante, y el acercamiento a la estructura factorial del cuestionario, de la que se presentan los datos más significativos, entre otros que la matriz de correlaciones cumple con las condiciones para su factorización; la medida de adecuación muestral de KaiserMeyer-Olkin se aproxima a 1 (.905) y la prueba de esfericidad de Bartlett resulta significativa (.000) con un alto valor de ji-cuadrado (23784.150). Por el procedimiento de componentes principales, se extraen ocho factores, siendo el más importante de ellos el de Estrategias de intervención en la comunidad.

El método de extracción elegido fue el de análisis de componentes principales y el de rotación ortogonal Varimax según Kaiser, de cara a acercarnos a la estructura simple de más fácil interpretación (Gil, 2003). Aplicando el criterio de Kaiser (1960) se han retenido los ocho que se pueden apreciar en la tabla 2, siendo el más importante de ellos el de Estrategias de intervención en la comunidad.

\section{TABla 2. Varianza total explicada por los ocho factores finalmente retenidos}

\begin{tabular}{cccc}
\multirow{2}{*}{ Componente } & \multicolumn{2}{c}{$\begin{array}{c}\text { Sumas de las saturaciones } \\
\text { al cuadrado de la extracción }\end{array}$} \\
\cline { 2 - 3 } & Total & $\begin{array}{c}\text { \% de la } \\
\text { varianza }\end{array}$ & $\%$ \\
\hline
\end{tabular}

\begin{tabular}{lrrr}
1 & 11.844 & 32,012 & 32,012 \\
\hline 2 & 3.369 & 9,106 & 41,118 \\
\hline 3 & 2.578 & 6,967 & 48,084 \\
\hline 5 & 1.611 & 4,355 & 52,439 \\
\hline 6 & 1.407 & 3,803 & 56,242 \\
\hline 7 & 1.282 & 3,464 & 59,706 \\
\hline 8 & 1.055 & 2,853 & 62,559 \\
\hline
\end{tabular}

Método de extracción: análisis de componentes principales.

Con la finalidad de facilitar la interpretación de los factores se acude a la denominada estructura simple, según Thurstone, para lo cual se procedió a obtener la matriz factorial rotada (tabla 3), mediante la que se pueden apreciar cuáles son las variables (ítems) con mayor peso en cada factor, lo que, una vez examinado el contenido 
de cada uno de ellos, facilita su comprensión y denominación. La tabla 3 muestra la denominada matriz $\mathrm{R}$ ordenada, en la que se eliminan aquellas variables con saturaciones inferiores a .35 para facilitar la interpretación.

\section{TABLa 3. Matriz rotada ordenada}

Factores*

\begin{tabular}{lllllllll}
\hline & 1 & 2 & 3 & 4 & 5 & 6 & 7 & 8 \\
\hline Ítems & & & & & & & & \\
\hline
\end{tabular}

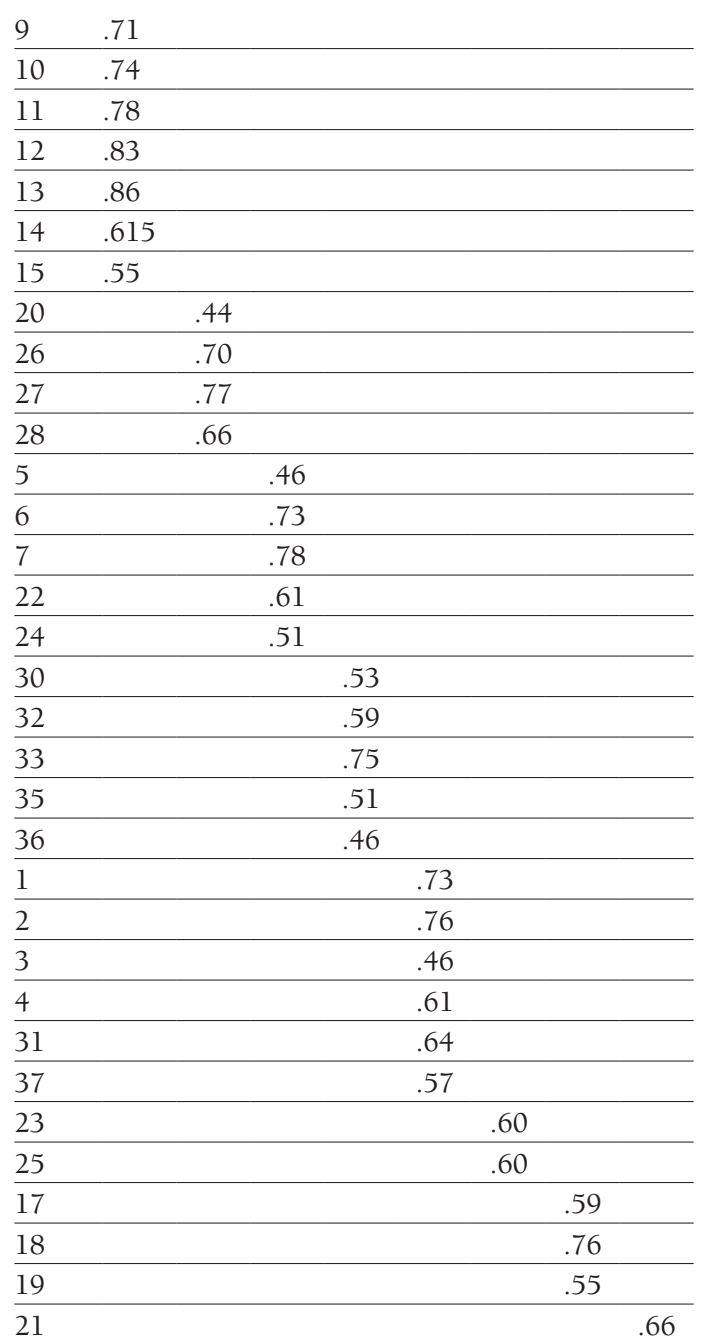

* Se han excluido las cargas factoriales <.35.
Como resumen de lo anterior, en la tabla 4 se presentan los ocho factores alcanzados, con indicación de la denominación atribuida y de los ítems que los saturan.

TABla 4. Resumen de factores del análisis factorial

Factor 1: estrategias de intervención en la comunidad

Ítems: b9, b10, b11, b12, b13, b14, b15. IFFS =.64

Factor 2: optimización de la intervención Ítems: c20, c26, c27, c28. IFFS =.44

Factor 3: estrategias de intervención individualizada Ítems: a5, a6, a7, c22, c24. IFFS =.53

Factor 4: orientación institucional

Ítems: c 30, c32, c33, c35, c36. IFFS $=.54$

Factor 5: función de apoyo o terapéutica

Ítems: a1, a2, a3, a4. IFFS $=.58$

Factor 6: coordinación con otros profesionales

Ítems: c23, c25, c31, c 37. IFFS $=.50$

Factor 7: optimización del trabajo comunitario

Ítems: b17, b18, b19. IFFS $=.49$

Factor 8: poco preciso

Ítem: c21. IFFS $=.23$

Del análisis de los datos llegamos a la conclusión de que, en propiedad, solo podemos hablar de una subescala aceptable empíricamente, la que define el primer factor, con valores de IFFS superiores a .6, con los ítems del 9 al 15 que explican el 32,012\% de varianza total. Estos ítems forman parte de la subescala B de trabajo comunitario, a la que en nuestro cuestionario añadíamos los ítems 16, 17, 18 y 19, todos, excepto el 16, en el factor siete que cuenta con valores de IFFS discretos. A nuestro juicio, no resulta inapropiado, dado su contenido, agruparlos en una subescala.

Por otra parte, los factores dos, cuatro, cinco $\mathrm{y}$ seis no presentan incompatibilidades con 
nuestras subescalas, ya que agrupan variables de forma coherente a las de orientación (A) y gestión (C) aunque el IFFS no es suficientemente elevado. Teniendo en cuenta que los 8 factores explican el 65,305\% de la varianza total podemos hablar de unos resultados que, en conjunto, resultan moderadamente compatibles en términos estadísticos con el modelo propuesto.

\section{Validez}

El proceso de validación se ha realizado a través de dos fases. Al confeccionar el CFES-R 2008 para su aplicación en el estudio de los Servicios Sociales, fue validado mediante el criterio de expertos. Estos validaron la redacción de los ítems, la claridad y la pertenencia de cada ítem a las funciones de la figura 1.

En una segunda fase, orientada al estudio sobre diversas áreas de intervención, adaptamos el anterior cuestionario, que pasó a denominarse CFES-R 2009. A tal efecto, se aplicó a un grupo piloto de 40 educadores en diferentes contextos profesionales (10 del área social, 10 que trabajaban con discapacitados, 10 con menores y 10 con adultos) todos ellos con cinco o más años de ejercicio profesional, a fin de ajustar su adecuación semántica y su pertinencia a los objetivos del mismo. Los resultados de dicho análisis se consideran satisfactorios por existir un consenso con la propuesta final del cuestionario, cuestión muy importante en el proceso de construcción.

\section{Juicio de expertos}

El cuestionario piloto CFES-Cuestionario para expertos5 fue sometido a juicio 20 expertos: 9 doctores (6 profesores universitarios de la UNED, dos de la Rovira y Virgili y uno de la Universidad de Castilla La Mancha) y 11 expertos técnicos (educadores sociales, trabajadores sociales y directores de servicios sociales, con más de cinco años de ejercicio profesional) para decidir sobre su validez de contenido. Valoraron la claridad en la redacción de los ítems, la suficiencia de ítems para medir las funciones genéricas y las tres subescalas del cuestionario, el grado de coherencia entre todos los elementos del mismo, la adecuación de las opciones de respuesta, la extensión del cuestionario y su diseño. Se incorporaron al instrumento los ítems que alcanzaban al menos un acuerdo del $80 \%$.

A partir del análisis de datos de los expertos, generamos la versión inicial CFES-R 2008. De las observaciones cualitativas se desprenden una serie de mejoras, que especificamos a continuación en la figura 3.

\section{Figura 3. Observaciones cualitativas de los expertos en CFES-R 2008}

Cambios en el CFES por observaciones cualitativas de los expertos

a) Es necesario priorizar el trabajo en menores pero también intervenir, detectar y orientar en situaciones de exclusión social, independientemente de la edad (ítems 1, 5 y 15)

b) El ítem 16 es muy parecido al 19, por lo que se suprime

c) Los ítems 29 y 30 son muy parecidos, por lo que se unifican en uno (el actual 29)

d) El actual 30 es un ítem nuevo a propuesta de los expertos: "Participación activa en formación y reciclaje profesional"

e) El anterior 26 se mejora en su redacción y se ubica en el apartado $B$, pasando a ser el $\mathrm{n}^{\circ} 16$

f) El 26 es un ítem nuevo surgido del proceso de validación: "Participación en la supervisión, contrastes y discusión de casos, metodología y proyectos de intervención"

g) Para finalizar, el ítem 37 hace referencia a la participación en procesos de contrataciones de personal se incluye por tratarse de una función que no nos aparece como genérica, por falta de consenso, y las puntuaciones bajas obtenidas contribuyen a comprobar la coherencia del cuestionario en el proceso de recogida de datos 


\section{Descriptivos del proceso de validación}

Junto a lo anterior se ofrece información analítica sobre los ítems, destacando el hecho de que todos ellos presentan un rango de medianas entre 3 y 5 y con las frecuencias acumuladas de las puntuaciones favorables de los expertos al instrumento ( $\geq 3$ ) entre el $75 \%$ y el $95 \%$. Los detallados en la tabla 5 son los que han sido objeto de revisión por presentar sus medianas valores $\leq 3$, en el $15 \%$ y más de las valoraciones de los expertos.

Globalmente constatamos que el cuestionario es razonablemente válido, ya que la mayoría de medianas de cada ítem es de 4 o 5, con la excepción del ítem 6 , que puntúa 3 y que ha sido profundamente modificado en atención a las sugerencias formuladas. Otro aspecto que se ha tenido en cuenta ha sido la frecuencia, ya que supone el grado de consenso y homogeneidad en las puntuaciones. En la figura 4 resumimos las aportaciones surgidas de todas estas valoraciones.

A partir de la aplicación a una muestra de 40 educadores, 10 por área de intervención, para ver las dificultades de implementación y adecuación semántica del cuestionario a los diversos contextos profesionales, realizamos los siguientes cambios para obtener el definitivo CFES-R 2009, reflejados en la figura 5.
FIgura 4. Diferencias entre CFES y CFES-R 2008

Resumen diferencias entre CFES y CFES-R 2008

Mejora de las instrucciones de implementación (p. 1) Introducción de tres bloques: A, B y C para ayudar a diferenciar las funciones

Funciones modificadas en el CFES-R 2008: A1, A5, A6, B13, B15, B16, C20, C26, C29. C30, C32, C37

\section{FIgURA 5. Diferencias entre CFES-R 2008 y CFES-R 2009}

Diferencias entre CFES-R 2008 y CFES-R 2009

Funciones 1 y 5 : suprimimos "priorizando menores". En la 5 añadimos "acompañamiento" — se indica como competencia en los nuevos planes de grado-

Función 2: reformulamos para comprensión más generalizada en "Recepción y análisis de las demandas de usuarios y familiares por parte de entidades, instituciones y otros servicios". Suprimimos "medio abierto" por ser un concepto restringido a Servicios Sociales

Funciones 14, 15, 16, 33 y 36: cambiamos "menores" por "usuarios"

Función 19: añadimos "profesionales"

Función 22: substituimos "segundo nivel" por "otros niveles"

Tabla 5. Descriptivos del proceso de validación

ÍTEM N Minimum Maximum Mediana Fa puntuaciones bajas*

\begin{tabular}{|c|c|c|c|c|c|}
\hline 5 & 20 & 1 & 5 & 4.5 & $25 \%$ \\
\hline 6 & 20 & 2 & 5 & 3 & $20 \%$ \\
\hline 13 & 20 & 1 & 5 & 5 & $15 \%$ \\
\hline 16 & 20 & 1 & 5 & 5 & $15 \%$ \\
\hline 20 & 20 & 2 & 5 & 4 & $20 \%$ \\
\hline 32 & 20 & 1 & 5 & 4.5 & $20 \%$ \\
\hline
\end{tabular}

$\mathrm{N}$ válidos 20

* Fa, se trata de la frecuencia acumulada de puntuaciones bajas (incluye valores $n=1$ y $n=2$ ). 


\section{Figura 5. Diferencias entre CFES-R 2008 y CFES-R 2009 (cont.)}

Diferencias entre CFES-R 2008 y CFES-R 2009

Función 24: reformulamos en "Seguimiento de la aplicación de recursos alternativos o complementarios". Suprimimos por lo que respecta a servicios del primer nivel ya que esta afirmación solo tiene sentido en los Servicios Sociales

Función 35: cambiamos "Servicios Sociales" por "nuestra institución"

Aplicación a un grupo piloto y contraste con las competencias específicas de Grado para confeccionar el CFES-R 2009

La desaparición de la diplomatura y la creación del Grado en Educación Social nos plantearon el problema de la adecuación del cuestionario para el segundo de los objetivos de nuestro trabajo.

Por ello, hemos realizado un acercamiento a la validación a partir de los estudios de Grado de Educación Social, que, necesariamente, deberá ser profundizado. Hemos contrastado las 20 competencias específicas establecidas por la Universidad Nacional de Educación a Distancia $(\mathrm{UNED})^{6}$ con nuestro cuestionario recogiendo el juicio técnico de tres profesores universitarios cuyo campo de especialización es el de los métodos de investigación y diagnóstico en educación. Hemos seleccionado la UNED por su ámbito nacional y por la dispersión de los profesionales que forma a través de todo el territorio. Sus valoraciones, recogidas en septiembre de 2011, quedan recogidas esquemáticamente en la figura 6, en cuyo interior aparecen los números de los ítems que tratan de valorar cada cruce de competencias y funciones.

De los resultados del mismo se desprende que todas las competencias de Grado se relacionan con las funciones genéricas que hemos definido en el proceso de validación, por lo que, en una primera aproximación y con la debida prudencia, consideramos que disponemos de un instrumento suficientemente válido para su utilización en este contexto.

\section{Fiabilidad de la escala}

El alfa de Cronbach permite cuantificar el nivel de fiabilidad de nuestro cuestionario sobre funciones generales del educador social, construido a partir de las 37 variables recogidas en el mismo. En particular, estas presentan mediciones estables y consistentes, con un elevado nivel de correlación entre ellas, como se desprende de los

FIGURA 6. Competencias específicas versus funciones del CFES-R 2009

\begin{tabular}{|c|c|c|c|c|c|c|c|}
\hline Comp. & Funciones & Comp. & Funciones & Comp. & Funciones & Comp. & Funciones \\
\hline 1 & $9,11,32,36$ & 6 & $20,28, \frac{32}{37}, 34,35$ & 11 & 5,9 & 16 & $\begin{array}{c}11,23,25,27, \\
28\end{array}$ \\
\hline 2 & $1,2,3,5$ & 7 & $3,8,11$ & 12 & 18 & 17 & 26 \\
\hline 3 & 3 & 8 & $19,26,27,28,31$ & 13 & 30,31 & 18 & 8 \\
\hline 4 & 10,13 & 9 & 11,18 & 14 & $\begin{array}{c}4,5,12,16,21,22 \\
23,24,25\end{array}$ & 19 & $10,11,17,33$ \\
\hline 5 & 11,13 & 10 & $13,29,34$ & 15 & $11,23,25,27,28$ & 20 & $14,20,30$ \\
\hline
\end{tabular}

Fuente: Guía del Grado en Educación Social de la UNED. 
datos de nuestra investigación. Las correlaciones entre ítem y total tienen un rango entre .381 y .671. Por otra parte, los coeficientes de fiabilidad obtenidos son: para el cuestionario global: .94; para las subescalas: .84 (A), .89 (B) y .90 (C), como observamos en la tabla 6 .

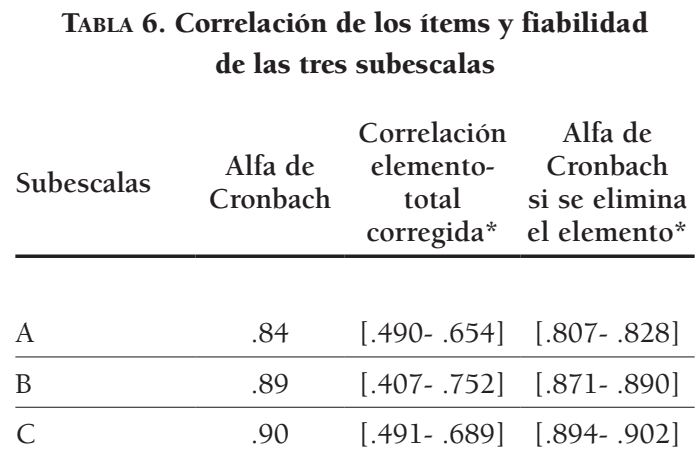

* Puntuaciones en intervalos globales de cada subescala.

En los tres casos se da una fiabilidad elevada, mayor de 8 , lo que es más que suficiente como garantía de fiabilidad de cada subescala. En nuestro cuestionario los valores de correlación de los ítems respecto a la puntuación total son superiores a .35 , con un nivel de significación de .01 o superior, excepto el ítem tres que puntúa 344.

En resumen: buenos índices de fiabilidad y correlaciones ítem-total superiores a .35, lo que representa un instrumento altamente fiable en general y en las subescalas.

\section{Discusión}

Nuestro instrumento para medir las funciones genéricas del educador social responde a un planteamiento de esta profesión en España; de hecho no hemos localizado, ni en nuestro país ni en otros, estudios empíricos, basados en cuestionarios, que atiendan a ese objetivo de la investigación. No obstante, a partir de la bibliografía relacionada, hemos constatado que el acompañamiento educativo que realizan estos profesionales es de suma importancia (Planella, 2003) como se deduce de nuestra investigación. También es relevante que el trabajo comunitario constituye una subescala empíricamente demostrada con valores IFFS superiores a .6; en este sentido, varios autores resaltan la importancia de la colaboración y el trabajo en equipo en los educadores (Green y Barnes, 1989; Kenaszchuk y otros, 2012; Petitpierre y otros, 2007).

En relación con otros trabajos realizados sobre esta profesión y con otros objetivos, nuestra investigación aporta un muestreo estatal y la recogida de datos, de profesionales representativos de los diferentes colegios profesionales en activo, contratados específicamente como educadores. En este aspecto coincidimos con Dixon y otros (2005) y Koster y Dengerink (2008) que dan gran importancia a las funciones realizadas en contextos laborales para su aplicación posterior a la formación de profesionales competentes.

\section{Conclusiones y prospectiva de investigación}

El Cuestionario CFES, una vez construido, validado, registrado ${ }^{7}$ y contrastado por su puesta en práctica, permite usos de innegable utilidad. Este cuestionario ha sido diseñado para identificar las funciones genéricas profesionales de los educadores en España. Si los datos empíricos aportados nos hacen pensar en un instrumento con razonable calidad, los datos disponibles desde su construcción nos alientan a seguir mejorándolo. En efecto: por una parte, contamos con datos sobre el elevado número de consultas del mismo y de sus resultados, expuestos en Vallés $(2009,2011)$ y en diferentes plataformas académicas y profesionales (se contabilizan más de 6.000 descargas).

A nuestro juicio, su utilidad fundamental consiste en reflejar globalmente las funciones genéricas de un contexto laboral o de un área de 
intervención. Una utilidad añadida, justamente en estos momentos en que ANECA está procediendo a la revisión de los planes de estudio, es la relativa al análisis de la relación existente entre la formación recibida y las funciones profesionales realmente ejercidas. Un ejemplo prospectivo del cuestionario es su uso a través de una tabla de especificaciones. Nuestro planteamiento, al respecto de esta tabla, se fundamenta en que las funciones profesionales se ejercen merced a los aprendizajes realizados y en relación a unas competencias adquiridas, por lo que se trata de una tabla coherente con nuestra investigación.

Dados nuestros planteamientos en torno a la relación entre la competencia profesional, las funciones que integran la profesión y la formación académica a los que nos hemos referido en la base teórica del cuestionario, y para dar la adecuada respuesta al segundo de nuestros objetivos - constatar la adecuación de la formación académica a la competencia profesional- se construyó una tabla de especificaciones en la que se relacionaban las asignaturas de la Diplomatura en Educación Social de la UNED con las competencias en las que debían ser formados los alumnos. En nuestro caso, en un eje, detallamos las asignaturas de la carrera de Educación Social, y en el otro, las competencias cristalizadas en los documentos de ANECA (2005), ASEDES (2006) y Senra (2006).

En la tabla de especificaciones, los números que aparecen en las intersecciones entre contenidos y competencias se refieren a los ítems destinados a medir las funciones, que pueden leerse en el cuestionario CFES-R 2009 que figura en el Anexo.

La tabla presentada nos permite proceder a la valoración de las asignaturas de la diplomatura (nos hemos fijado en los resultados de aprendizaje) y su análisis con base en nuestro instrumento. Veámosla en los anexos.

Por último, queremos resaltar la conveniencia de una nueva validación directa y detallada abordando la relación de cada uno de los ítems con las competencias del grado y con las funciones profesionales ejercidas en diversos contextos. Esta nueva validación permitiría confirmar, en el caso de ser positiva, su validez en el contexto profesional y académico actual, ampliando el último trabajo llevado a cabo por los tres expertos universitarios, tomando como referencia las competencias del Grado de Educación Social en la UNED.

\section{Notas}

${ }^{1}$ Las conclusiones de este estudio las publicamos en la parte $1^{\text {a }}$ del libro Manual del educador social. Intervención en Servicios Sociales, año 2009, editorial Pirámide.

${ }^{2}$ Derecho de la ciudadanía que se concreta en el reconocimiento de una profesión de carácter pedagógico, generadora de contextos educativos y acciones mediadoras y formativas, que son ámbito de competencia profesional del educador social, posibilitando:

- La incorporación del sujeto de la educación a la diversidad de las redes sociales, entendida como el desarrollo de la sociabilidad y la circulación social.

- La promoción cultural y social, entendida como apertura a nuevas posibilidades de la adquisición de bienes culturales, que amplíen las perspectivas educativas, laborales, de ocio y participación social.

${ }^{3}$ Para definir los contextos laborales mayoritarios partimos de la investigación de Cacho (1996), siendo estos del área social, del menor, de atención a personas con discapacidad y atención a personas adultas (incluye a mayores).

${ }^{4}$ La mayoría de encuestados no cuestiona la existencia de funciones genéricas aunque, por algunas respuestas cualitativas, apreciamos en el 10\% de los casos de nuestra muestra cierta resistencia a contestar según nuestra propuesta. 
${ }^{5}$ Cuestionario de análisis de las funciones del Educador (-a) Social en los Servicios Sociales de Atención Primaria en Cataluña: CFES. Ejemplar de validación. Disponible en: <http://pepvalles.blogspot.com.es/2013/11/blog-post.html>

${ }^{6}$ Competencias específicas del grado de Educación Social de la UNED disponibles en: http://portal.uned.es/portal/ page?_pageid=93,24265331\&_dad=portal\&_schema=PORTAL\&idContenido=3

${ }^{7}$ El CFES consta, como obra científica, en el Registro de la Propiedad Intelectual de la Generalitat de Cataluña con el $n^{o}$ de asiento registral 02/2012/2599.

\section{Referencias bibliográficas}

ANECA (2004). Título de grado en pedagogía y educación social. Recuperado de http://www.aneca. es/var/media/150392/libroblanco_pedagogial_0305.pdf >

Arandia, M., Fernández-Fernández, I., Alonso, M. J., Uribe-Etxebarria, A., Beloki, N., Ramiro, A., Aguire, N., y otros (2012). Formación y desarrollo profesional de los educadores sociales en el ámbito de la infancia desprotegida: contexto y perspectivas en la comunidad autónoma vasca, Revista de Educación, 359. Septiembre-diciembre 2012, 505-529.

ASEDES (2004). Hacia una Definición de Educación Social. Recuperado de https://www.google.es/

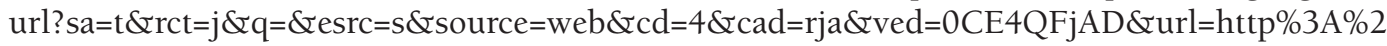
F\%2Fwww.eduso.net\%2Fred\%2Fdefinicion.htm\&ei=t6KKUtmGKMPfswaX64GoCw\&usg=AF QjCNHSA8cZfOlkrJ48XFqpp5JLd7in0A\&sig2=NFOKp5bDNQgjBYP6J_5nvg

ASEDES (2006). Catálogo de funciones y competencias. Recuperado de https://www.google.es/url $? \mathrm{sa}=\mathrm{t} \& \mathrm{rct}=\mathrm{j} \& \mathrm{q}=\& \mathrm{esrc}=\mathrm{s} \&$ source=web $\& \mathrm{~cd}=1$ \&ved $=0$ CDQQFjAA $\& u r l=h \mathrm{ttp} \% 3 \mathrm{~A} \% 2 \mathrm{~F} \% 2 \mathrm{Fwww}$. eduso.net\%2Farchivos\%2Ffuncionesycompetencias.doc\&ei=R6KKUpziNo_FswbJw4DgCg\&us g=AFQjCNET26dJ6YU0Ba65gjQO8UIgUjaOcA\&sig2=j-_srr02tAI4HgkgoPctg\&bvm=bv.56643 336,d.Yms

Cacho, X. (1996). L'educadora i l'educador social a Catalunya. Barcelona: APESC.

Calderón, M. J., y Gotor, V. (coords.) (2013). La profesión de la educación social en Europa. Estudio comparado. Barcelona: Consejo General de Colegios de Educadoras y Educadores Sociales (CGCEES).

Caride, J. A. (2002). Construir la profesión: la educación social como proyecto ético y tarea cívica. Pedagogía Social: Revista interuniversitaria, 9, 91-125.

Caride, J. A. (2005). Las fronteras de la Pedagogía Social: perspectivas científica e histórica. Barcelona: Gedisa.

Dixon, R., Meier, R. L., Brown, D. C., y Custer, R. L. (2005). The Critical Entrepreneurial Competencies Required by Instructors from Institution-Based Enterprises: A Jamaican Study. Journal of Industrial Teacher Education 42.4, 25-51. Recuperado de: http://scholar.lib.vt.edu/ejournals/JITE/ v42n4/dixon.html

Gil, J. A. (2003). Métodos de Investigación en Educación. Vol. III: Análisis Multivariante. Madrid: UNED.

Green, J. E., y Barnes, D. L. (1989). Do Your "Aides" Aid Instruction? A Tool for Assessing the Use of Paraprofessionals as Instructional Assistants, Teacher Educator, 24 (3), 2-9.

Guerrero, E., y Calero, J. (2013). El aprendizaje basado en proyectos como base metodológica en el grado de Educación Social, Educación social. Revista de Intervención Socioeducativa, 53, 73-91.

Kenaszchuk, C., Rykhoff, M., Collins, L., McPhail, S., y Van-Soeren, M. (2012). Positive and Null Effects of Interprofessional Education on Attitudes toward Interprofessional Learning and Collaboration, Advances in Health Sciences Education, 17 (5), 651-669. doi: 10.1007/s10459-0119341-0 
Korncbeck, J. (2002). Reflections on the exportability of social pedagogy and its possible limits, Social Work in Europe, 9 (2).

Koster, B., y Dengerink, J. J. (2008). Professional Standards for Teacher Educators: How to Deal with Complexity, Ownership and Function. Experiences from the Netherlands, European Journal of Teacher Education, 31 (2), 135-149. doi: 10.1080/02619760802000115

López de Aróstegui, R. (1995). El perfil profesional del Educador y la Educadora Social en Euskadi. Vitoria: Gobierno Vasco.

Moyano, S. (2012). Acción educativa y funciones de los educadores sociales. Barcelona: UOC.

Múgica, J. (1991). Imagen del educador desde la Asociación Agintzari. Bilbao: Geuk - Asociación de Educadores Especializados.

Oficina Europea de la Asociación Internacional de Educadores Sociales (2005). Plataforma común para las educadoras y los Educadores sociales en Europa. Barcelona: Diputación de Barcelona.

Pérez, G. (2004). Pedagogía Social. Educación Social. Construcción científica e intervención práctica. Madrid: Narcea.

Petitpierre, G., Lobo, D., Dietrich, A., Benz, M., y Adler, J. (2007). Integration of Education and Care Given to Children with Profound Multiple Disabilities in Switzerland, Journal of Policy and Practice in Intellectual Disabilities, 4 (2), 141-151.

Petrus, A. (1993). Educación social y perfil del educador social. En J. Sáez (coord.), El educador social (pp. 165-214). Murcia: Universidad de Murcia.

Petrus, A. (1997). Concepto de Educación Social. En A. Petrus (ed.), Pedagogía Social, (pp. 9-39). Barcelona: Ariel.

Planella, J. (2003). Fonaments per a una pedagogia de l'acompanyament en la praxi de l'educació social, Revista Catalana de Pedagogia, 2, 13-33.

Quintana, J. M., Montoya, J. M., y Lebrero, Mª P. (2002). Pedagogía Social. Madrid: UNED.

Romans, M. (2000). Funciones y competencias del Educador Social. En M. Romans, A. Petrus y J. Trilla, De profesión: educador (a) social (pp. 151-185). Barcelona. Paidós.

Ruby, M (2012). The Role of a Grandmother in Maintaining Bangla with Her Granddaughter in East London, Journal of Multilingual and Multicultural Development, 33 (1), 67-83.

Sáez, J. (2003). La profesionalización de los educadores sociales: en busca de la competencia educativa cualificadora. Madrid: Dykinson.

Sáez, J., y García, J. (2006). Pensar en la Educación Social como profesión. Madrid: Alianza Editorial.

Scarpa, P., y Corrente, M. (2007). La dimensión europea del educador social. En Revista de Pedagogía Social - SIPS, no 14, tercera época, 63-65.

Senra, M. (2006). Valoración de las competencias profesionales específicas del Educador Social. En E. de Lara y J. Quintanal (coords.), El prácticum en las titulaciones de Educación: Reflexiones y experiencias (pp. 135-142). Madrid: Dykinson.

Senra, M. (2012). La formación práctica en intervención socioeducativa. Madrid: UNED/Sanz y Torres.

Sierra, R. (1988). Técnicas de investigación Social. Teoría y Ejercicios. Madrid: Paraninfo.

Tiana, A., y Sanz, F. (2003). Génesis y situación de la educación social en Europa. Madrid: UNED.

Utley, B., y Rapport, M. K. (2002). Essential Elements of Effective Teamwork: Shared Understanding and Differences between Special Educators and Related Service Providers. Physical Disabilities: Education and Related Services, 20 (2), 9-47. Recuperado de: http://files.eric.ed.gov/fulltext/ EJ842018.pdf

Vallés, J. (2009). Manual del educador social: intervención en los servicios sociales. Madrid: Pirámide. 
Vallés, J. (2011). Análisis y valoración de las funciones del educador en España (tesis doctoral). Madrid: UNED. Recuperado de http://e-spacio.uned.es/fez/view.php? pid=tesisuned:EducacionJvalles

Varela, L. (2012). La Educación Social en las políticas públicas de bienestar: programas, experiencias e iniciativas pedagógico-sociales en los servicios sociales comunitarios (tesis doctoral). Santiago de Compostela: USC. Recuperado de http://dspace.usc.es/handle/10347/8169

\section{Anexos}

\section{Cuestionario de análisis de las funciones del Educador(-a) Social en España}

Instrucciones para su administración:

Antes de responder al cuestionario lea atentamente las siguientes instrucciones:

1. Objetivo de este cuestionario es analizar las funciones del Educador (a) Social en diversas áreas de actuación: social, personas adultas, menores o discapacitados en España. Se pretende discriminar las funciones más importantes.

2. Destinatarios: Está dirigido a los educadores sociales o habilitados que estén contratados como educadores que trabajen en las áreas: social, personas adultas, menores o discapacitados, en España.

3. Instrucciones para contestar el cuestionario:

3.1. Comience completando los datos personales para posteriormente responder al cuestionario — si lo contesta con el ordenador se le activarán las opciones en X o numéricas al clicar encima de la casilla-.

3.2. El cuestionario está pensado para rellenar de diversas formas (a su elección):

a) por separado y de forma independiente por un educador

b) por varios educadores del equipo conjuntamente

3.3. Cada una de las afirmaciones del cuestionario describen las funciones óptimas que deben poseer los/ as educadores/as. Marque con una "X" la casilla correspondiente según la frecuencia o intensidad con la que se cumple esa afirmación en su equipo. Responda de acuerdo con la siguiente escala:

1: Nada

2: Muy poco

3: Algo

4: Bastante

5: Mucho

3.4. Se ruega responda a todos los ítems. No existen respuestas verdaderas o falsas. Puede realizar los comentarios que considere oportunos a las cuestiones planteadas en el apartado final de sugerencias 
I. Datos personales y profesionales

Tipología del encuestado:

Tipología de trabajo

Marque con una cruz su lugar de trabajo. Si se considera de estas áreas de actuación pero su lugar de trabajo no figura puede contestar en "otros" y especificarlo a continuación :

Otros:...
Educador/a

Equipo (especificar $n^{\circ}$ de educadores del equipo)

\section{Área social:}

Prevención de la exclusión social, la violencia y las toxicomanías

$\square$ Rehabilitación de toxicomanías

$\square$ I nstituciones penitenciarias

$\square$ Instituciones de bienestar social

$\square$ Atención a la familia

$\square$ Centros de acogida a mujeres maltratadas

$\square$ Asociaciones de usuarios y consumidores

$\square$ Programas de atención y orientación a inmigrantes

$\square$ Otros

Área de educación de personas adultas:

Centros de día

$\square$ Centros de educación de personas adultas

$\square$ Residencias para personas adultas

$\square$ Hogares de jubilados

$\square$ Programas para la inserción laboral de la mujer

$\square$ Otros

\section{Área del menor:}

Centros de reforma de menores conflictivos

$\square$ Hogares protegidos para menores

$\square$ Residencias para menores

$\square$ Otros

Área de atención a personas con discapacidad:

Residencias específicas para personas con discapacidad

$\square$ Asociaciones de ayuda a personas con discapacidad

$\square$ Centros Ocupacionales

$\square$ Centros Especiales de Empleo

$\square$ Otros

Comunidad autónoma donde ejerce la profesión:

Años de experiencia como educador/a: 


\section{Cuestionario de análisis de las funciones del Educador(-A) Social en España, CFES-R 2009}

\section{A) En relación con el trabajo individual y grupal}

\begin{tabular}{|c|c|c|c|c|c|}
\hline & 1 & 2 & 3 & 4 & 5 \\
\hline $\begin{array}{l}\text { 1. Detección y prevención de situaciones de riesgo o de exclusión social de } \\
\text { los usuarios y sus familias. }\end{array}$ & $\square$ & $\square$ & $\square$ & $\square$ & $\square$ \\
\hline $\begin{array}{l}\text { 2. Recepción y análisis de las demandas de usuarios y familiares o por parte } \\
\text { de entidades, instituciones y otros servicios. }\end{array}$ & $\square$ & $\square$ & $\square$ & $\square$ & $\square$ \\
\hline $\begin{array}{l}\text { 3. Elaboración, seguimiento y evaluación del plan de trabajo educativo } \\
\text { individual y, si procede, en coordinación con otros profesionales o } \\
\text { Servicios del primer nivel. }\end{array}$ & $\square$ & $\square$ & $\square$ & $\square$ & $\square$ \\
\hline $\begin{array}{l}\text { 4. Información y orientación al individuo y a su familia, de las prestaciones } \\
\text { y los recursos sociales del territorio que puedan facilitar la intervención } \\
\text { educativa. }\end{array}$ & $\square$ & $\square$ & $\square$ & $\square$ & $\square$ \\
\hline $\begin{array}{l}\text { 5. Aplicación y evaluación del tratamiento educativo de apoyo y } \\
\text { acompañamiento en personas y/o familias que tienen especiales } \\
\text { dificultades en su proceso de socialización. }\end{array}$ & $\square$ & $\square$ & $\square$ & $\square$ & $\square$ \\
\hline $\begin{array}{l}\text { 6. Apoyo a la familia y al trabajador social en la tramitación y seguimiento } \\
\text { de algunas prestaciones individuales o ingresos en centros educativos y/o } \\
\text { terapéuticos. }\end{array}$ & $\square$ & $\square$ & $\square$ & $\square$ & $\square$ \\
\hline $\begin{array}{l}\text { 7. Tramitación de propuestas de derivación a los servicios de atención } \\
\text { especializada, de acuerdo con el resto de miembros del equipo, así como } \\
\text { su seguimiento y el posterior apoyo a los procesos de reinserción social }\end{array}$ & $\square$ & $\square$ & $\square$ & $\square$ & $\square$ \\
\hline $\begin{array}{l}\text { 8. Elaboración de informes socio-educativos para finalidades protectoras, } \\
\text { preventivas, terapéuticas y ocasionalmente asistenciales. }\end{array}$ & $\square$ & $\square$ & $\square$ & $\square$ & $\square$ \\
\hline
\end{tabular}

B) En relación con el trabajo comunitario

\begin{tabular}{|c|c|c|c|c|c|}
\hline & 1 & 2 & 3 & 4 & 5 \\
\hline $\begin{array}{l}\text { 9. Detección y prevención de situaciones de riesgo social o de exclusión } \\
\text { de grupos con necesidades de intervención social, concretándolas en } \\
\text { proyectos de prospección, sensibilización y dinamización. }\end{array}$ & $\square$ & $\square$ & $\square$ & $\square$ & $\square$ \\
\hline $\begin{array}{l}\text { 10. Recepción y análisis de las demandas provenientes de entidades o de } \\
\text { grupos vecinales. }\end{array}$ & $\square$ & $\square$ & $\square$ & $\square$ & $\square$ \\
\hline $\begin{array}{l}\text { 11. Promoción, elaboración y seguimiento de los proyectos de intervención } \\
\text { comunitaria con colectivos de personas afectadas con las mismas } \\
\text { problemáticas sociales. }\end{array}$ & $\square$ & $\square$ & $\square$ & $\square$ & $\square$ \\
\hline $\begin{array}{l}\text { 12. Información y orientación de los recursos y servicios sociales que } \\
\text { permitan paliar las limitaciones de la comunidad a través de la } \\
\text { utilización de medios de comunicación y estrategias comunicativas. }\end{array}$ & $\square$ & $\square$ & $\square$ & $\square$ & $\square$ \\
\hline $\begin{array}{l}\text { 13. Promoción de la organización y/o animación de la comunidad para } \\
\text { conseguir una mejora en el nivel social, y posibilitar la resolución de los } \\
\text { problemas. }\end{array}$ & $\square$ & $\square$ & $\square$ & $\square$ & $\square$ \\
\hline 14. Sensibilización y educación ciudadana en relación con nuestros usuarios. & $\square$ & $\square$ & $\square$ & $\square$ & $\square$ \\
\hline $\begin{array}{l}\text { 15. Recogida de datos y elaboración de propuestas de futuros recursos para } \\
\text { nuestros usuarios y planificación global del sector. Participación en } \\
\text { propuestas para otros colectivos con marginación social. }\end{array}$ & $\square$ & $\square$ & $\square$ & $\square$ & $\square$ \\
\hline 16. Selección de los usuarios para cada proyecto. & $\square$ & $\square$ & $\square$ & $\square$ & $\square$ \\
\hline
\end{tabular}


17. Elaboración de un fichero actualizado tanto de datos individuales como de proyectos.

18. Intervención en las situaciones conflictivas con finalidad educativa (función mediadora), siempre que las condiciones sean las adecuadas y lo requiera alguno de los casos.

19. Contacto y coordinación con profesionales, grupos y/o entidades con la finalidad de optimizar las actuaciones.

\section{C) En relación con el trabajo en equipo y los encargos institucionales}

\begin{tabular}{lllll}
\hline & 1 & 2 & 3 & 4 \\
\hline $\begin{array}{l}\text { 20. Valoración de las necesidades del servicio en referencia a la mejora de la } \\
\text { intervención. }\end{array}$ & $\square$ & $\square$ & $\square$ & $\square$ \\
\hline $\begin{array}{l}\text { 21. Coordinación con el resto de los servicios del mismo nivel, tanto para } \\
\text { el establecimiento de criterios y prioridades, como para el trabajo } \\
\text { individual y familiar. }\end{array}$ & $\square$ & $\square$ & $\square$ & $\square$ \\
\hline
\end{tabular}

22. Derivación de los casos a los Servicios de otros niveles.

23. Seguimiento conjunto con el diplomado de trabajo social del equipo en los procesos de inserción.

24. Seguimiento de la aplicación de recursos alternativos o complementarios.

25. Evaluación conjunta con el diplomado de trabajo social del equipo de los resultados globales de la intervención efectuada.

26. Participación en la supervisión, contraste y discusión de casos, metodología y proyectos de intervención.

27. Valoración continua de la aplicación de los proyectos para la elaboración de la memoria final.

28. Vigilancia de los plazos establecidos en los proyectos que impulsen.

29. Promoción del trabajo en red, en los proyectos que impulsen, asegurando la participación de todos los agentes sociales de forma ágil y con los apoyos que se precisen.

30. Participación activa en formación y reciclaje profesional.

31. Asistencia y soporte técnico a los monitores o trabajadoras familiares que realicen la intervención directa.

32. Orientación a los servicios e instituciones sobre aspectos educativos comunitarios vinculados a la propia intervención.

33. Recopilación de información sobre actividades y entidades relacionadas con nuestros usuarios para optimizar recursos y planes educativos.

34. Información, a los representantes de los ayuntamientos y entidades, de la aplicación de los programas comunitarios impulsados por el equipo de educadores.

35. Proposición de adaptaciones o reformas constructivas en nuestra institución para mejorar la intervención.

36. Defensa de los derechos de los usuarios a nivel institucional y cuando se detecte algún posible maltrato institucional.

37. Participación en procesos de selección de personal en relación a plazas de educadores/as u otros técnicos relacionados con proyectos que se vayan a coordinar. 
Sugerencias para mejorar el cuestionario CFES-R 2009

Sugerencias de mejora de los ítems que contiene:

Sugerencias de incorporar otros ítems o funciones o eliminar alguno:

Otros comentarios:

Observaciones:

El cuestionario consta de 37 ítems y de cinco páginas en total. Por favor, revise que no haya ítems en blanco.

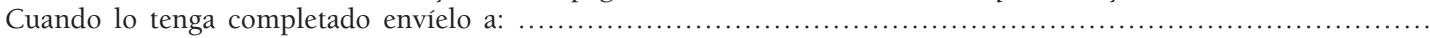
Si tiene dudas llame al teléfono ..................................... Le agradecemos su colaboración, que contribuirá a conocer mejor nuestra profesión. 


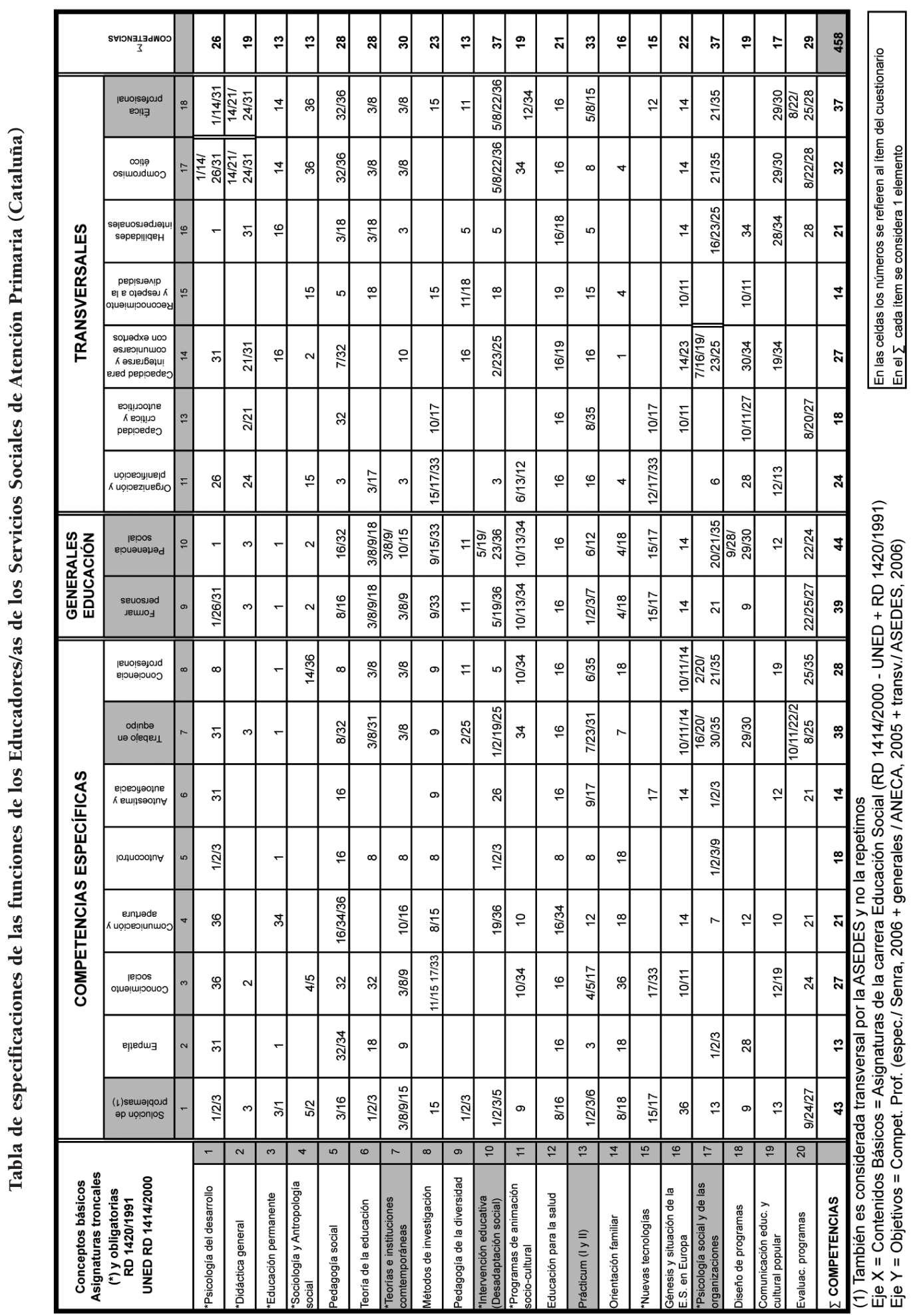


Abstract

The role of the social educator: validation of the CFES-R questionnaire

INTRODUCTION. This article presents the CFES-R questionnaire, on the role of social educators (known in the English-speaking countries, as social workers or community workers), as a useful and adequate instrument to study, evaluate and research on the generic functions of their job. It contains those generic educational functions that work effectively for individual and social wellbeing of vulnerable children and socially excluded people or those at risk of exclusion. Additionally, we test its use as an instrument to evaluate the relation between the education and training received by the workers by means of a specification table. METHOD. A descriptive and correlational research has been carried out, on a nationwide sample of 1242 workers from 16 different regions (Autonomous Communities). The instrument, prepared according to the classical test theory, has been made up by developing a theoretical model based on the therapeutic or retraining assistance to the beneficiaries, differentiating three subscales of roles, namely: counselling, community work and management roles. RESULTS. The questionnaire achieves high reliability in Cronbach's alpha test and shows sufficient validity obtained from professional documents and through expert panels. The overall reliability is .94 and those of the subscales (personal counselling, community work and management) are around .90. DISCUSSION. The 37 items of the instrument show stable and consistent measurements. The functions about the retraining assistance are relevant and the existence of a subscale of community functions is proven. Further studies are required for other subscales (counselling and management). The instrument could help to assess the academic training and to review and improve the curriculum.

Keywords: Job descriptions, Functions, Educators, Community education, Questionnaires, Reliability, Validity, Therapy.

\section{Résumé}

Les fonctions de l'educateur social specialisé: questionnaire de validation CFES-R

INTRODUCTION. Dans cet article, est présenté le Questionnaire sur le rôle de l'Éducateur Social Spécialisé (CFES-R) comme instrument approprié et à la fois utile pour l'étude, l'évaluation et la recherche sur les fonctions génériques de la profession. Son contenu comprend les fonctions éducatives génériques qu'exercent les professionnels de l'éducation de manière efficace en faveur du bien-être individuel et social des enfants et des personnes vulnérables socialement exclues ou en risque d'exclusion. En outre, étant donné la relation nécessaire entre la formation académique et la formation reçue pour l'exercice compétent de fonctions professionnelles, est reflétée sur un tableau de spécifications son utilisation possible comme outil pour les évaluer. MÉTHODE. On a réalisé une étude descriptive et corrélativement, avec un échantillon de 1.242 éducateurs dans les 16 régions ('Communautés Autonomes'/'Comunidades Autónomas' de l'Espagne). Linstrument auquel on a recourt pour son élaboration et validation est celui de la théorie classique des tests; il a été construit à partir du développement d'un modèle théorique basé sur le soutien thérapeutique ou de réadaptation aux bénéficiaires, en distinguant trois sous-échelles de fonctions: guidage, communautaire et gestion. RÉSULTATS. Le questionnaire permet d'obtenir une fiabilité élevée, mesurée avec l'alpha de Cronbach et a une validité de contenu suffisant obtenue à 
partir de documents professionnels élaborés par des groupes d'experts. La fiabilité globale est de .94 et la des sous-échelles (orientation personnelle, travail communautaire et gestion) autour de .90. DISCUSSION. Les 37 items de l'instrument ont des mesures stables et cohérentes. Les fonctions dans leur soutien rééducatif sont pertinentes et prouvent l'existence de la sous-échelle des fonctions communautaires. Les deux autres sous-échelles (orientation et gestion) exigent de poursuivre des études plus approfondies. Il peut être un instrument très utile dans l'évaluation de la formation académique et même, à la révision et à l'amélioration des programmes d'étude.

Mots clés: Les descriptions de poste de travail, les rôles, les éducateurs, l'éducation communautaire, les questionnaires, la fiabilité, la validité, thérapeutique.

\section{Perfil profesional de los autores}

\section{Josep Vallés Herrero (autor de contacto)}

Profesor tutor de Educación Social y Trabajo Social de la UNED. Doctor en Pedagogía. Coordinador de Extensión Universitaria y de la UNED Senior en el centro de Tortosa. Miembro de la Sociedad Iberoamericana de Pedagogía Social. Presidente del Colegio de Educadoras y Educadores Sociales de Cataluña - CEESC - en "Terres de l'Ebre". Miembro del Consejo Asesor de la Facultad de Educación y Psicología de la Universidad Rovira i Virgili de Tarragona. Ha participado en dos proyectos de investigación (I+D) de la UNED. Es profesor colaborador del curso de Enseñanza Abierta de la UNED "Intervención socioeducativa con menores" y vocal del Observatorio de los Derechos de la Infancia de la Generalitat de Cataluña.

Correo electrónico de contacto: jvalles@tortosa.uned.es

Dirección para la correspondencia: UNED Centro Asociado de Tortosa. Calle Cervantes, 17, 43500 Tortosa, Tarragona.

\section{Ramón Pérez Juste}

Catedrático emérito de la UNED. Ha sido director del Departamento de Métodos de Investigación y Diagnóstico en Educación de la UNED; decano de la Facultad y vicerrector de Ordenación Académica y Profesorado. Ha desempeñado el puesto de presidente de la Sociedad Española de Pedagogía, director de Bordón y vicepresidente del Consejo Escolar del Estado. Director de más de 30 tesis doctorales, trabaja temas de metodología de la investigación y evaluación de programas e instituciones educativas. Director de diez proyectos de investigación subvencionados. En la actualidad dirige el Máster en Liderazgo y Dirección de Centros Educativos.

Correo electrónico de contacto: rperez@edu.uned.es 